issn: $1808-799 \mathrm{X}$

ano 5 - número 5 - 2007

artigo

\title{
DE LA EDUCACIÓN COMO “CAPITAL HUMANO” A LA EDUCACIÓN COMO “ACCOUNTABILITY": UN SINUOSO PERIPLO QUE SEÑALA LOS CAMBIOS EN LA RELACIÓN EDUCACIÓN, ECONOMÍA Y TRABAJO EN LAS SOCIEDADES CAPITALISTAS.[I]
}

\author{
R.Gerardo Bianchetti[ii] \\ gerbian@unsa.edu.ar \\ Universidad Nacional de Salta
}

\section{INTRODUCCION}

El aprendizaje de una actividad que suponga cambio o modificación de un determinado objeto (natural o creado por el ser humano) requiere de saberes que relacionan múltiples dimensiones de las capacidades humanas y que implican además, alguna forma de vinculación con el medio natural y/o social.

Desde el aprendizaje por imitación, al aprendizaje de los modernos lenguajes que ponen en acción procesos automatizados de la producción industrial, las característica del trabajo adquirió cada vez mayor complejidad a la vez que requería, crecientemente, de conocimientos especializados y/o formalizados. 
Como requerimiento derivado de esas transformaciones, las sociedades fueron llevadas a crear y generalizar de manera ampliada, formas institucionales destinadas a transmitir los saberes considerados necesarios para su reproducción y continuidad histórica.

La Historia de la Educación ha permitido conocer las diferentes respuestas que las sociedades elaboraron para cumplir con esos objetivos, quedando en evidencia que las características de las instituciones educativas, tienen relación directa con el nivel de complejidad de las estructuras sociales.

En cada etapa histórica de las sociedades y como consecuencia de los procesos que fueron causas de las transformaciones sociales, a la transmisión de valores y principios relacionados con la convivencia social, se le sumaron nuevas funciones derivadas de la necesidad de legitimar un orden político - social, como así también, los conocimientos y habilidades prácticas, requeridos por el sistema de producción e intercambio dominante.

Desde los inicios de la modernidad y hasta la actualidad, la educación incorporó una parte de los saberes sociales como contenidos escolares. Las necesidades políticas, de la economía o los intereses ideológicos de los distintos grupos, sectores o clases sociales, disputaron su influencia para la formación de los sujetos sociales, con el objetivo de naturalizar un determinado orden social.[iii]

Por estas razones la educación se convirtió en un ámbito de disputas, las que se expresaron tanto a nivel de las teorías, como en las formas concretas como se materializaban las mismas.

Una de las relaciones que adquirió importancia creciente durante el siglo XX es la que vincula a la educación con la economía y el trabajo, en la medida que los procesos económicos requerían formaciones especializadas para responder a las necesidades del modelo de desarrollo.

El estudio de esa articulación abre muchas perspectivas de análisis, en la medida que entre los analistas del tema no existe unanimidad sobre el contenido atribuido a los 
términos que lo constituyen. Considerar que la educación debe formar "para el empleo" o "para el trabajo"; para adaptarse a las "demandas del mercado" o a la "participar en la construcción de un proyecto de desarrollo independiente"; que constituye un capital adquirido por las personas o una inversión productiva de las empresas, etc. desencadena un amplio debate ya que esas consideraciones derivan de matrices teóricas distintas y expresan además modelos sociales diferentes.

\section{Y EN EL PRINCIPIO ... FUE GRATUITA Y OBLIGATORIA}

Los análisis que utilizan una variable histórica, comprueban que, en las sociedades capitalistas la relación educación - trabajo - economía, reconoce varias etapas.

En los primeros tiempo, es el trabajo el que adquiere centralidad como consecuencia de que

"se constituye por primera vez en el medio privilegiado de integración social: en el contexto socioeconómico de la industrialización y de la división técnica que ella provoca, el trabajo aparece como el mejor medio de encontrar un lugar, su lugar, en la sociedad. La ideología del trabajo, progresivamente, se apoderó de todas las mentes e impuso su condición de normalidad'[iv].

Un autor, que expresa las ideas de la época, al describir los problemas de la educación en las sociedades europeas del siglo XIX[v] destaca que

“(la) crisis universal de la enseñanza tiene un marcado carácter económico. Se propone, ante todo, tácita o expresamente, desarrollar las aptitudes de trabajo del individuo y mejorar las condiciones de la sociedad. Sin disfraces ni símbolos religiosos y éticos, la firme y cruda preocupación de la riqueza parece predominar sobre otras preocupaciones".[vi]

El paulatino reconocimiento de la función económico - social que comienza a adquirir la educación en las sociedades capitalistas, se tradujo en el desarrollo de teorías que trataban de explicar de qué manera y a través de qué mecanismos era posible sellar esa vinculación, como también, y en sentido contrario, dio lugar al surgimiento de elaboraciones críticas a esas formulaciones. 
Un representante de la primera vertiente teórica, sostenía que

"es la sociedad la que, para poder subsistir, necesita que el trabajo se reparta entre sus miembros y se reparta entre ellos de tal forma y no de tal otra. Este es el motivo por el cual la sociedad se preocupa de preparar, a través de la educación, los trabajadores especializados de quienes está necesitada".[vii] (Durkheim)

La integración social por medio del trabajo, suponía el aprendizaje de conocimientos técnico-prácticos como también la adquisición de comportamientos adecuados a la disciplina existente en el ámbito laboral.

Compayré, en el "Curso de Pedagogía" y que es una obra que muestra las ideas pedagógicas de finales del siglo XIX resalta la importancia que se le daba a las adquisición de "costumbres", entendidas como formas automatizadas de comportamientos. En sus propios términos:

"la educación no es, en gran parte, más que el arte de formar buenas costumbres (... de inteligencia, sentimiento y acción)", las que se adquieren con la repetición de los actos dado que "unas proceden de las inclinaciones y de los instintos; otras de actos reflexivos a los que ha colaborado la voluntad. La misión del educador es pues vigilar, ya los instintos, ya las primeras manifestaciones de la voluntad'.

La repetición de los comportamientos debía ser gradual hasta lograr su naturalización: "contentémonos al principio con que la realice con indiferencia, para que la repita después con placer hasta formar la costumbre. Insinuemos, en una palabra, y no impongamos las costumbres".

El trabajo de los educadores era importante para esta tarea ya que, según su opinión, "depende de nosotros que la costumbre no sea más que un modo cómodo de hacer sin esfuerzo lo que hemos hecho anteriormente con reflexión y voluntad. La costumbre consolida la obra de la libertad."[viii]

En el mismo sentido de argumentación, Amancio Alcorta sostenía que

"la democracia, si ha de ser tan benéfica como puede serlo, requiere de cada persona la aptitud indispensable para proceder en las relaciones humanas en armonía con los principios morales; para aplicar al trabajo diario, sea de la clase que se quiera, las leyes económicas; para realizar el derecho como más 
conviene a la naturaleza del hombre; requiere un alto grado de saber, no sólo de las ciencias técnicas, sino también de las ciencias morales y jurídicas, porque son sus conclusiones los fundamentos del orden de las sociedades_públicas y privadas". [ix]

En estas afirmaciones se pone de manifiesto la intención de lograr la convergencia entre principios morales, leyes económicas y naturaleza humana, y además señala el camino por el que se supone, ha de transitar la educación para desarrollar las "aptitudes personales".

En todas estas opiniones se destaca el énfasis en el carácter integrador al "mundo social" que se le reconocía a la educación, como también ponen en evidencia, cómo en las primeras etapas en el desarrollo de los sistemas educativos nacionales esa preocupación estaba referida, de manera prioritaria, a la adquisición de comportamientos que facilitaran la adaptación social, en todas sus dimensiones, ya que esas actitudes tendrían efecto tanto en el ámbito familiar, como en las demás prácticas sociales.

En esas primeros momentos, la "gratuidad" de la educación constituye la expresión de una voluntad política, que consideraba deber del Estado contribuir a garantizar una instrucción general que serviría para instalar y mantener el orden social. Como señala Puelles Benitez:

"La Constitución (francesa) de 1791 en su subtítulo I garantizará el establecimiento de una instrucción pública, común a todos los ciudadanos, gratuita respecto de aquellas partes indispensables para todos los hombres." [x]

La obligatoriedad se amplía bajo la influencia jacobina ya que en la Declaración de Derechos del Hombre y del Ciudadano (1793) se hace referencia a la instrucción en general y no solamente a la elemental.

“Obligatoriedad y Gratuidad” de la educación se han de convertir en una decisión política destinada a construir consensos alrededor de un determinado proyecto político y en consecuencia se ha de transformar en uno de los principales ejes del debate contemporáneo, referido a los alcances de la responsabilidad del Estado en el sostenimiento de esos postulados, enfrentando los valores y principios liberales con los provenientes de matrices teóricas con mayor sentido social. 
Esa preocupación se mezcla también con aquellas opiniones, que en esas primeras etapas del desarrollo capitalista, dejaban en claro que algunos trabajos no requerían de instrucción escolar. Taylor, uno de los teóricos, junto con Fayol, de la organización científica del trabajo, al referirse a las cualidades requeridas para alguno de ellos sostenía que

"uno de los requisitos para el hombre, que es apropiado para tener como ocupación la de manejar hierro en lingotes, es que ha de ser tan estúpido y flemático, que en su conformación mental ha de parecerse más a un buey que otro tipo de ser'[xi]

La voluntad política y la opinión de los "especialistas" van a terminar coincidiendo, aunque con un cierto grado de refinamiento retórico, en los fundamentos de las teorías que justificaban la "necesaria diferenciación" entre "trabajo manual e intelectual". Esa división de tareas,

"reduce a unos al puro aspecto teórico de planificación y a otros al ámbito práctico - mecánico. La fragmentación del trabajo afecta tanto a los unos como a los otros; pero en lo concreto legitima el traslado del "saber" del trabajador a la gerencia, a la vez que abarata, en términos relativos, el costo - y el valor - del trabajador y lo reduce a una somnolencia intelectual vitalicia".[xii]

Cuando esta división de funciones es analizada desde una perspectiva crítica se la considera como una "alienación" a la que es obligado el trabajador, por ser un mecanismo necesario a la reproducción del modo de producción capitalista. Trasladada al ámbito de las decisiones de las políticas educativas se materializa en las funciones que se le asignaban a los diferentes niveles del sistema, el que a través de las modalidades canaliza las necesidades y objetivos del poder político (formar la clase dirigente / disciplinar al ciudadano), como también las funciones requeridas por el sistema productivo (conducir / obedecer ; concebir / ejecutar ).

En esta primera etapa de la historia de nuestras sociedades, la influencia de las teorías evolucionistas, el determinismo biologicista del positivismo o los resabios de un orden social estamental, vigente en el pasado cercano, favoreció la hegemonía de 
creencias que legitimaban, desde el sistema educativo formal, la jerarquización de la sociedad, pese a la resistencia que opusieron las corrientes igualitaristas que intentaron, a través de la educación no formal, difundir sus ideas.

La paulatina y creciente relación entre educación, economía y trabajo iniciada en estas primeras etapas del desarrollo capitalista, va a ser definida como la "perspectiva instrumentalista y funcional de la educación",[xiii] por el hecho que estudia la vinculación entre las demandas de la economía y las funciones asignadas al sistema educativo, con el objetivo de favorecer la reproducción de las relaciones sociales capitalistas.

Los desarrollos teóricos del siglo XIX, surgidos desde posiciones críticas al capitalismo, no abordaron en los primeros momentos y de forma directa, el análisis de la relación entre la educación y el mundo del trabajo, sin embargo:

"las ideas al respecto se encuentran diseminadas a lo largo de varios de sus escritos (de Marx y Engels), manteniéndose en una misma línea durante más de cuatro décadas. En esos escritos la problemática educativa está planteada de modo ocasional, fragmentario, pero siempre en el contexto de la crítica de las relaciones sociales y de las líneas maestras de su modificación"[xiv].

La crítica marxista al "modo de producción" capitalista constituye una forma de análisis que propone una interpretación relacional de los hechos sociales.

"Para Marx, el sujeto se constituye como resultado de su implicación en la producción social de su propia existencia material, a través de las que llama relaciones sociales y técnicas de producción".[xv]

En esta concepción, el trabajo no constituye una actividad "técnica" sino un engranaje más del sistema social. La perspectiva relacional de los procesos sociales, utilizada para explicar la vinculación entre educación y trabajo, muestra las implicaciones que poseen ambas dimensiones.

La preocupación por cuestiones como la enajenación en el trabajo corresponde, según algunos autores, al "Marx joven", es decir cuando todavía formaba parte de la izquierda hegeliana.[xvi] El "Marx maduro" escribirá "El Capital” preocupado por la crítica de la economía política y su teoría del valor...".[xvii] A partir de ambas experiencias, esta 
matriz teórica aportó sólidos argumentos para demostrar la vinculación entre filosofía, economía y política.

El interés por desentrañar los mecanismos ocultos que explican la vigencia de un determinado sistema económico - social, impulsó a los pensadores de esta corriente, a preocuparse por buscar las respuestas detrás de las apariencias, contribuyendo, de esa manera, a desarrollar en el ámbito de las ciencias sociales, una actitud de búsqueda permanente alejada, de las posiciones dogmáticas y deterministas. (No siempre algunos de sus adherentes pudieron evitar caer en estas últimas conductas)

La formación "para y en el trabajo", se constituyó en un principio fundamental de esta teoría en relación con las funciones de la educación, aunque la forma y los objetivos se diferencian claramente de la lógica, los principios y valores que posee en las sociedades capitalistas.[xviii]

Estos desarrollos teóricos aportaron nuevos elementos para comprender a la educación como fenómeno político, cuestionando en consecuencia, su supuesta neutralidad.

El trabajo como "alienación" en la sociedad capitalista y el trabajo como liberación del hombre de las limitaciones y condicionamientos impuestos por la naturaleza, conviven en los análisis generados desde esta matriz teórica, la que se constituyó en un componente ineludible del debate educativo contemporáneo.

Y LUEGO LA ECONOMIA . . . . . DESCUBRE A LA EDUCACION ...

"La característica más común del futurólogo económico no es la de saber, sino la de no saber que no sabe. Su máxima ventaja es que todas las predicciones, acertadas o inexactas, se olvidan con rapidez".[xix]

\section{Waldo Ansaldi nos muestra cómo el concepto}

\section{"Democracia", puede estar asociado a un número muy}




\title{
importante de adjetivos :
}

" A guisa de ejemplo, señalo una lista nada exhaustiva: burguesa, capitalista, de baja intensidad, delegativa, directa, formal, liberal, limitada, nominal, obrera, participativa, popular, protegida, radical, real, representativa, restringida, revolucionaria, social, socialista, sustantiva, tutelada... apenas una veintena de adjetivos entre no menos de iquinientos!!.[xx]

Una polisemia similar se puede encontrar en referencia al "trabajo". Como describe un autor:

\begin{abstract}
"el trabajo se revistió, ciertamente, con los ropajes más diversos y dispares. El trabajo como único fundamento de la riqueza de las naciones y de la felicidad personal de sus ciudadanos; el trabajo como actividad fundadora de la conciencia de uno mismo, de la pertenencia a una sociedad y de la alteridad respecto a la naturaleza, el trabajo como trampa de la alienación individual y social, como medio imprescindible para la recuperación de la autenticidad personal y para el desarrollo de la conciencia crítica que abre el camino a la liberación social; el trabajo como expresión de espiritualidad religiosa y como categoría inexcusable de la teología de las realidades mundanas; el trabajo como manifestación de la virtud patriótica del ciudadano nacionalizado; el trabajo como dispositivo privilegiado para la configuración del hombre motivado, movido por los deseos e intereses a los que el trabajo promete satisfacción; el trabajo como garantía de la autonomía y de la libertad personales; el trabajo como realización de las capacidades creativas o simplemente habilidosas e industriosas, de unos seres humanos perspicaces e inteligentes, a las que sistemáticamente retroalimenta; el trabajo como actividad esforzada que satisface moralmente como deber cumplido; el trabajo como lazo de socialización y de sociabilidad de los que lo comparten y, en general, de los que se necesitan para la realización de una obra o la prestación de un servicio; el trabajo como conformador de léxicos específicos facilitador de relaciones dialógicas con un alto nivel de implicaciones de los hablantes, el trabajo como referente básico de la solidaridad social; el trabajo como arma para la liberación de colectivos discriminados u oprimidos . . . [xxi]. ... y podrían ser más ...
\end{abstract}

La variada combinación que es posible establecer entre las distintas consideraciones sobre el trabajo constituye uno de los soportes de los modelos sociales, como así también define los alcances y/o límites que se le reconocen a la educación en esa relación.

Los sentidos asignados al "trabajo", pueden demandar a la educación funciones 
diferentes: algunos pondrían énfasis en los contenidos, en las relaciones pedagógicas, en los valores, en los modelos institucionales, en las formas y límites de vinculación con la sociedad, en la adquisición de habilidades prácticas, etc. como también en la combinación de muchas de ellas.

En la primera parte de este trabajo, intentamos presentar cómo en las primeras etapas del desarrollo industrial de las sociedades capitalistas, la educación cumplía, principalmente, funciones moralizantes y disciplinadoras de las conductas sociales, ya que las habilidades necesarias para el trabajo se adquirían en el ámbito laboral.

En ese momento histórico, el discurso pedagógico y las políticas educativas en la Argentina reflejaban esta tendencia. La obligatoriedad (y por consiguiente la responsabilidad del Estado) se limita a la "instrucción básica”. La formación en los niveles superiores del sistema pasaría a ser una decisión personal o de la familia.

Amancio Alcorta, muestra la preocupación de la época sobre la necesidad de que la educación colabore en el disciplinamiento de los comportamientos, al afirmar que :

"La instrucción es un derecho del individuo, del padre de familia. No hay discusión posible a este respecto. Pero la instrucción es también indispensable para el orden social, para la garantía recíproca de los intereses privados".[xxii]

Si bien es posible reconocer que la relación educación - economía - trabajo adquiere importancia creciente, los estudios que vinculan esta relación con el desarrollo económico comienzan a generalizarse a mediados del siglo XX, cuando se introduce el concepto de "capital humano", como situación que explica las diferencias de ingreso en las personas, como así también en relación con el desarrollo económico de las sociedades. Ese concepto, concebido desde la perspectiva social del liberalismo, es utilizado para demostrar que el nivel educativo alcanzado por el individuo, es expresión de la inversión económica que cada uno está dispuesto a realizar sobre sí mismo[xxiii]. De acuerdo a uno de los teóricos más importante de esta corriente

"El componente de la producción derivado de la instrucción, es una inversión en habilidades y conocimientos que aumenta futuras ingresos y de ese modo se asemeja a una inversión en (otros) bienes de producción (Schultz, $T$. 1962)[xxiv]. 
Para "Coleman y sus colaboradores, no era la escuela sino el alumno el factor esencial en la determinación del éxito o el fracaso escolares."[xxv]

Esta relación utilizada en los análisis macroeconómicos, servía para explicar (y justificar) las diferencias en el desarrollo económico de las sociedades. Aumento de la "productividad" individual y aumento de la "renta nacional" son, desde esta óptica, las dos caras de la misma moneda.

La creencia en que las situaciones individuales eran las que determinaban en gran medida los logros alcanzados, situaba el eje de los análisis de estas teorías, en el ámbito de la familia y no en la escuela o en el contexto social.

Como nos recuerda Guerrero Serón

“...en 1972 Ch. Jenks y M.J. Bane vinieron a confirmar que la escuela no es responsable de las desigualdades sociales y no las cambia, estableciendo que el origen y el entorno familiares son los principales factores en el éxito escolar y laboral y que las reformas educativas sólo aportan variaciones menores a las desigualdades de partida".[xxvi]

El optimismo asociado a la relación "mayor educación - mejora económica ascenso social", se favorece por las condiciones derivadas de la reconstrucción de los países occidentales luego de la Segunda Guerra Mundial y que se los ha calificado como "los treinta gloriosos". Esa recuperación económica, en Europa, contó con la ayuda estadounidense a través del Plan Marshall y sirvió para reactivar la economía en ambos continentes.

Las políticas económicas tanto en Europa como en los EEUU se fundamentaron en los aportes teóricos Keynesianos y aunque EEUU fue refractario a las estrategias regulacionistas, los economistas de esa época adherían a las ideas de generar pleno empleo para recompensar, según algunas interpretaciones, a los trabajadores que habían sufrido los daños producidos por la guerra[xxvii].

Las convergencia de estos factores facilitaron la movilidad social y la educación adquirió un destacado reconocimiento social. 
Si bien la teoría del capital humano recibió cuestionamientos teóricos[xxviii] por no haber quedado demostrado que siempre se cumple la correspondencia entre educación, productividad y crecimiento económico, logra incorporarse al discurso político como fundamento de las propuestas que promueven esa vinculación, pudiendo introducir el cálculo especulativo, propio de la economía liberal, como principio y valor social.

Por otro lado, en la medida que el crecimiento económico favorecía la mejora en las condiciones de vida de una cantidad variable de personas, en las diferentes sociedades, la educación se instaló en el "sentido común"[xxix] de amplios sectores sociales como el vehículo que garantizaba esa movilidad social, expectativa que, concretada en algunas etapas, se mantuvo independientemente de los cambios operados en la economía.

La teoría del capital humano sostiene que la educación constituye un capital que el individuo adquiere en los distintos niveles del sistema educativo, y por lo tanto es la expresión de una voluntad de inversión económica, que cada individuo se propone realizar para alcanzar mejores condiciones de vida

Según esta interpretación, las demandas sobre las responsabilidad que le cabe al Estado en el sostenimiento de un sistema educativo obligatorio y / o gratuito queda limitada a la atención de los sectores sociales más pobres, ya que son las voluntades individuales, o de la familia del resto de los sectores sociales, las que definirán los accesos y permanencia en el sistema educativo.

La voluntad política que impulsaba la "Obligatoriedad y gratuidad" a finales del siglo $\mathrm{XIX}$, pasa a ser considerada como la expresión de un intervencionismo del Estado que atenta contra la libertad de elección en el primer caso, y en el segundo, como una injusticia ya que pone en evidencia de los desequilibrios que provoca el Estado cuando reemplaza la ley de la libre competencia, con decisiones consideradas compulsivas.

\section{Y EN LOS '90... LA EDUCACION SE CONVIERTE EN UNA INVERSION}

Las transformaciones económicas y sociales que comienzan a producirse en Europa a mediados de la década de los '70, y que se las define como reformas "neoliberales", se 
imponen, a la mayoría de los países subdesarrollados de América Latina, a través de las políticas monitoreadas desde algunos organismos multilaterales y a las que adherían grupos de poder económico y político locales.

Estas reformas, que basaban sus propuestas en la teoría económica liberal, y en los principios y valores del conservadurismo social y político, pretenden generalizar la lógica y el cálculo especulativo que rige el intercambio en el mercado, a todas las prácticas sociales.

Mientras en el siglo XIX y gran parte del XX, en relación con la educación, se reconocía la importancia del Estado como instancia mediadora entre los intereses públicos y privados, aunque responsable de garantizar el equilibrio que suponen las leyes formales; las reformas de los ' 90 buscaron retirarlo de esas funciones para facilitar la expresión "espontánea" de las "leyes económicas".

La estabilidad de las políticas neoliberales requería de un cambio en la filosofía de las instituciones sociales, que surgieron o se desarrollaron en vinculación estrecha con la estructura funcional del Estado y esos cambios suponían también de modificaciones en los comportamientos sociales, incorporando o fortaleciendo los valores que faciliten el desarrollo y naturalización del nuevo proyecto social.

Las críticas a las funciones desempeñadas por el Estado, a lo largo de su historia contemporánea, estaban dirigidas a sus actividades como: propietario de las empresas de servicios públicos; regulador de la economía; redistribuidor de recursos económicos; proveedor de servicios de protección social; etc.

En la Argentina, la propuesta de establecer un "Estado Mínimo" se asemejó en los hechos, a la idea de un "Estado Infimo", encargado de crear, prioritariamente, las mejores condiciones para la reproducción ampliada del capital.

Las reformas educacionales de inspiración neoliberal, se presentaron dentro de un amplio espectro de alternativas, las que en el discurso se asociaba con estrategias de modernización social. Escuelas de autogestión, financiamiento con bonos educativos, 
escuelas "charter", flexibilización de los controles sobre las instituciones privadas, sistemas de gerenciamiento propio de las empresas, fueron algunos de los proyectos impulsados en algunas jurisdicciones provinciales y que tenían en común el hecho que, de forma explícita o implícita, primaba la lógica económica.

La diferencia entre esta nueva perspectiva teórica que analiza la relación educación economía y trabajo y la que surgió a mediados del siglo XX radica en el hecho de que en aquella, tal como lo expresamos precedentemente, la educación representaba un capital de las personas que les permitía acceder a mejores trabajos y mayores salarios, en la nueva interpretación de la relación, la educación se convierte en un factor de producción, pero no asociado a las personas sino a las estrategias financieras que permiten una rápida y segura reproducción del capital.

Los cambios introducidos en el mundo por la "globalización neoliberal" afectan todas las dimensiones de las prácticas sociales y en consecuencia las instituciones existentes en esas sociedades, sufren el impacto de los proyectos que responden a los nuevos modelos institucionales.

Entre los estudios que analizan la hegemonía neoliberal en esta etapa histórica, existe coincidencias en considerar que las estrategias políticas se subordinan a los intereses del capital, que promueve la lógica y los principios del "mercado" a todas las formas de relación social. La economía se transforma así en "la ciencia social” que dispone de los fundamentos y el método, adecuado para regir los comportamientos sociales.

El lenguaje social se ve invadido por conceptos provenientes del campo de la economía o que tienen fuertes connotaciones relacionadas con ella. Competencia, competitividad, calidad, eficacia, eficiencia, productividad, gestión, gerenciamiento, etc. son algunos de los más utilizados, sin embargo es también en los valores que se promueven, donde se percibe el sentido utilitarista propio de ese modelo social.

En el ámbito de la educación, la influencia de estas ideas se fortalece con las políticas que llevan adelante algunos organismos internacionales los que mediante el 
financiamiento de programas, imponen cambios en las características y funcionamiento de los sistemas educativos.

Sin embargo, es quizás en la idea de introducir la "accountability" en la educación, dónde se percibe con mayor claridad la nueva filosofía.

Según sus promotores

"No existe una sola palabra que sirva para traducir "accountability" al español. El concepto puede describirse como "responsabilidad por resultados", "fijación de responsabilidad" o "rendición de cuentas". El término proviene de la esfera financiera y describe la responsabilidad que le cabe a las personas a quienes se confía dinero". [xxx]

De acuerdo con esta propuesta, los resultados logrados por las instituciones, puede servir como indicio a los padres (consumidores) sobre la calidad de la oferta (imagen y desempeño institucional) con el objetivo de evaluar la conveniencia o no de "adquirir el producto".

La competencia se establece tanto a nivel interinstitucional, como también al interior de las instituciones a partir de la competencia de "talentos" entre los propios docentes, que son quienes han de otorgar prestigio a la propuesta educativa.

Estos nuevos escenarios tienden a conformar un "cuasi mercado", el que según sus defensores

"no se refiere solo al aspecto relacionado con la obtención de lucro por parte de las unidades educativas, sino con una organización competitiva del sistema educativo y de su asignación de recursos, aun cuando el uso de los mismos sean sin fines lucrativos". [xxxi]

Mientras que para Witty, analizando estas propuestas aplicadas en otros países asegura que

"cada vez se utiliza más la expresión "cuasimercado" para caracterizar estos intentos de introducir las fuerzas del mercado y las formas de decisión propia del sector privado en la provisión de la educación y de los servicios de bienestar".[xxxii]

En un intento por precisar más el concepto destaca que 
"Levacic señala que las características distintivas de un cuasimercado en relación con un servicio público, son la separación entre comprador y proveedor y un elemento de elección del usuario entre distintos proveedores. En otras palabras, la provisión del servicio se separa de su financiación, de manera que distintos proveedores puedan competir por la prestación del servicio". (Idem)

En la actualidad, la idea de que nos encontramos frente a la "sociedad del conocimiento", sirve de incentivo a las personas en la búsqueda por apropiarse de las herramientas más adecuadas para acceder a los mejores puestos laborales. Esta necesidad favorece la dinámica de la empresa capitalista, concebida y organizada para "responder a la demanda".

La educación se convierte en un insumo más de la cadena productiva y un terreno para las inversiones, principalmente en los niveles superiores del sistema educativo ya que son los que, por las características de los sectores sociales demandantes, garantiza mayores ingresos económicos.

\section{CONCLUSIONES (siempre provisorias)}

En las etapas de organización de los Estados Nacionales, la "instrucción", considerada como el instrumento fundamental para crear los vínculos sociales necesarios para conformar una identidad, se convirtió en una "razón de Estado" y en consecuencia, la obligatoriedad de formación en el nivel básico del sistema expresaba una voluntad política que priorizaba la estabilidad social.

A mediados del siglo XX, economía y educación establecen una relación de correspondencia, en la que la primera se favorece con los aportes provenientes de la mayor productividad de los "recursos humanos" formados en las instituciones educativas y por el desarrollo tecnológico derivado de esa mayor y mejor formación.

Por otra parte, los sujetos sociales a través de la educación incrementan sus capacidades (teórico - prácticas) mejorando su desempeño laboral y permitiendo un aumento en sus ingresos y una mejora en su condición social.

En las etapas de expansión económica, esta relación pudo ser constatada lo que 
sirvió para fortalecer esa correspondencia.

Dentro de esa misma lógica, algunas variaciones, como las teorías "meritocráticas" relativizaban algunos supuestos enfatizando sobre otros (origen social, capital social y/o cultural, etc) sin cuestionar los aportes de la educación al sistema productivo.

A finales del siglo XX y como consecuencia de los cambios tecnológicos, las características del nuevo capitalismo, el reagrupamiento político de los Estados, el desarrollo de la ciencia, etc, la relación entre educación, economía y trabajo, sufre una reformulación que incide sobre las decisiones políticas, de los países capitalistas, que se orientan a consideraciones de tipo cualitativo más que cuantitativo.

La economía globalizada, impone una organización empresaria que necesita nuevas capacidades (teórico-prácticas) mientras que la educación formal reduce su influencia. La producción basada en la "oferta" demanda una formación que se adapte a los múltiples requerimientos de un mercado ampliado, diversificado y en algunos casos "sofisticado".

En ese contexto la educación deja de ser exclusivamente un "capital humano", para convertirse también en un "capital del capital" que es quien define y establece las condiciones para utilizar la educación como "valor de cambio".

Las teorías que incorporan los criterios de la "accountability" en la educación introducen la perspectiva empresaria en la evaluación de los resultados de la educación. Esta evaluación se realiza en dos direcciones, la primera se refiere al ámbito personal: cada uno debe asumir los resultados de su trabajo y la segunda se vincula a lo institucional: grado de afectación de las acciones sobre las demás instituciones (familia, otras instituciones formadoras, etc), que les provee información para decidir entre las diferentes ofertas.

En ambos casos la evaluación de las responsabilidades se mide a través de una escala de valores. Para quienes adhieren a esta idea, los valores se asocian creando una "cadena de valor" que 
"vincula de forma lógica actividades deseables que al ser alineadas y ejecutadas crean un nuevo valor mayor que la suma del valor de las actividades individuales'[xxxiii].

En esa cadena, "todo lo que se piensa y se hace - las políticas y las prácticasnecesitan alinearse y ser consistentes con estos valores"

Estas afirmaciones dejan ver, por un lado, cómo se reconoce la necesaria vinculación que debe existir entre las diferentes prácticas sociales a través de la convergencia de valores, y por otro, cómo el "valor mayor" subordina los individuales para lograr la estabilidad social.

El logro de los objetivos de la educación se mide por los "estándares" alcanzados y este concepto hace referencia a "lo que deben saber los alumnos en distintas asignaturas y en los distintos niveles de escolaridad'. [xxxiv]

La definición de esos contenidos surge de la delimitación de los principios y valores considerados necesarios para "(preparar) a los jóvenes para tener éxito y bienestar como adultos en los mundos del trabajo, la familia y la ciudadanía".[xxxv]

La utilización de referentes de la economía en las cuestiones vinculadas a procesos educativos favorece y facilita la hegemonía de un sistema de valores propios del capitalismo de competencia. La construcción de un "sentido común" que utiliza un mismo lenguaje para referirse a cuestiones cualitativamente distintas, tiende a favorecer a quienes se benefician con la instauración de ese modelo social.

Las estrategias del neoliberalismo en el ámbito de la educación muestran el nuevo rostro del capitalismo en el inicio del nuevo siglo.

Mientras en el pasado el interés político, por medio de la educación gratuita y obligatoria se preocupaba por universalizar la formación básica para formar ciudadanos y disciplinados trabajadores, en la actualidad la influencia del ideario neoliberal sobre la política, impulsa la tendencia a formar buenos y educados consumidores.

\section{BIBLIOGRAFIA}


ALCORTA, A. (1886) “La Instrucción Secundaria” La Cultura Argentina. Bs. As. (Reedicc. 1916)

ANSALDI W. (2007) "La democracia en América Latina, un barco a la deriva”. Pág. 37.FCE. Bs.As

BUNGE, Carlos .O. (1928) "La Educación”. Libro I. Espasa -Calpe. S.A. Madrid

COMPAYRÉ G. (1904) "Curso de Pedagogía". Librería de la Vda.de Ch. Bouret. Paris

CORVALÁN, J. (2006) "Accountability educacional. CIDE - PREAL. Santiago. Chile

DIEZ, F. (2001) "Utilidad, deseo y virtud. La formación de la idea moderna de trabajo". Ediciones Península. Barcelona España FRIGOTTO. G. (1989) "A produtividade da escola improdutiva". Cortez Editora. Sao Paulo. Brasil. GALBRAITH, J.K. (1991) "Historia de la Economía". Pág. 14. Ariel Sociedad Económica. Bs.As

GIL VILLA, F. (1994) “Teoría sociológica de la educación”. Amarú Ediciones. Salamanca. España GUERRERO SERÓN, A. (2003) "Enseñanza y Sociedad” Siglo XXI. España.

HOPENHAYN,M. (2006) "Repensar el trabajo". Grupo Editorial Norma. Bs.As

MANNO, F (2006) "Accountability educacional:posibilidades y desafíos para América Latina a partir de la experiencia internacional". CIDE - PREAL. Santiago. Chile

MCMEEKIN,R.W. (2006) "Hacia una comprensión de la Accountability educativa y cómo puede aplicarse en los países de América Latina". En "Accountability educacional...

PALACIOS ,J.(1997) “La educación en el siglo XX.”.Ed.Laboratorio Educativo. Venezuela.

PORTELLI, H. (1997) “Gramsci y el bloque histórico”. Siglo XXI. México.

PUELLES BENITEZ, M. (1993) "Estado y Educación en el desarrollo histórico de las sociedades europeas" Revista Iberoamericana de Educación Nro. 1. Madrid. España

TENTI FANFANI, E. (2004) "Sociología de la Educación”.Universidad Nacional de Quilmes. Bs.As

WHITTY, POWER y HALPIN (1999) “La escuela, el estado y el mercado”. Ed. Morata. Madrid

[i] El trabajo es resultado de análisis realizados para el Proyecto de Investigación PAV 2003 - 180 "Intersecciones entre desigualdad y educación media- un análisis de las dinámicas de producción y reproducción de la desigualdad escolar y social en cuatro jurisdicciones". Directora Ines Dusell

[ii] Profesor de Historia de la Educación y Política Educacional Argentina. Univ.Nacional de Salta. (Argentina)

[iii] Tenti Fanfani, E. 
[iv] Hopenhayn,M.(pág 122).

[V] "El siglo XIX, como he dicho, es el siglo de la educación, no sólo por su conquista de nuevos métodos y por la democrática difusión de la enseñanza, sino también por las grandes crisis de su instrucción pública. La antinomia entre las prácticas todavía escolásticas y las teorías ya empíricas de la enseñanza en la Edad Moderna engendra en la Edad contemporánea una franca lucha de la instrucción científica y de lenguas vivas contra el clasismo tradicional"'” Bunge, Carlos .O.

[vi] Bunge. C. (op.cit.pág. 109).

[vii] Gil Villa, F. (pág. 36).

[viii] Compayré G. (pág. 221 y ss).

[ix] Alcorta, A.

[x] Puelles Benítez, (pág. 37)

[xi] Hopenhayn M. ( op.cit. pág 147)

[xii] Idem (pág 151)

[xiii] Un muy buen análisis sobre este tema: Frigotto. G.

[xiv] Palacios ,J.

[xv] Idem (pág 45)

[xvi] Guerrero Serón, A.. (pag. 37 y ss) "Se trata de tres manuscritos, elaborados en 1844, sobre cuestiones de economía y filosofía, con planteamientos muy ligados aún a la izquierda hegeliana y a Feuerbach que permanecieron ocultos hasta 1932, en que su publicación transformó en gran medida el pensamiento marxista.

[xvii] Idem.

[xviii] Palacios, J. op.cit.

[xix] Galbraith, J.K.(pág. 14).

[xx] Ansaldi W. (pág. 37)

[xxi] Diez, F.

[xxii] Op. cit. (pág. 25)

[xxiii] Gil Villa. op.cit.

[xxiv] Frigotto, G. ( op.cit.pág.40). (traducción propia)

[xxv] Guerrero Serón, A. (op.cit. pág 178)

[xxvi] Guerrero Serón. (op. cit. pág. 180). 
[xxvii] Galbraith, K. (op. cit. pág. 276 y ss)

[xxviii] "Una de las críticas más completas e interesantes a la teoría del capital humano, es la de L.C.Thurow". en Gil Villa, op.cit.

[xxix] Se utiliza este concepto en los términos que le asigna Gramsci, es decir como "una amalgama de diversas ideologías tradicionales y de la ideología de la clase dirigente" ..."esta concepción del mundo se presenta bajo una multiplicidad de formas: "su rasgo más fundamental y más característico es de ser una concepción disgregada, incoherente, conforme a la posición social y cultural de las multitudes, cuya filosofía es". Portelli, $\mathrm{H}$.

[xxx] McMeekin,R.W.

[xxxi] Corvalán, J.(op.cit. Pág. 17).

[xxxii] Whitty, y otros.

[Xxxiii] Manno, F (op.cit. (pág. 53)

[xxxiv] McMeekin (op. cit. pág. 21)

[xxxv] Manno, F. (op.cit. pág 57) 